\title{
Salvage Hyperthermic Gemcitabine and Docetaxel Combination Chemotherapy After BCG Failure in Non-Muscle Invasive Bladder Cancer Patients
}

\author{
Mounica Y. Rao ${ }^{\mathrm{a}}$, Paul Kang ${ }^{\mathrm{a}}$, Jamaka C. Tarajkowski ${ }^{\mathrm{b}}$, Debra L. Mobley ${ }^{\mathrm{b}}$ and Donald L. Lamm ${ }^{\mathrm{a}, \mathrm{b}, *}$ \\ ${ }^{\mathrm{a}}$ University of Arizona College of Medicine- Phoenix, Phoenix, AZ, USA \\ ${ }^{\mathrm{b}}$ BCG Oncology, Phoenix, AZ, USA
}

Received 13 August 2019

Accepted 4 November 2019

\begin{abstract}
.
BACKGROUND: Bacillus Calmette-Guerin (BCG) is the recommended therapy for high and intermediate risk non-muscle invasive bladder cancer (NMIBC), but treatment failure is common. While a radical cystectomy is recommended after BCG failure, some patients desire bladder preservation and others are poor surgical candidates. Salvage chemotherapy treatments may be offered to this subgroup of patients.

OBJECTIVE: To assess if combination, hyperthermic Gemcitabine and Docetaxel chemotherapy (GEM/DOCE) is a safe and effective salvage option for treating NMIBC.

METHODS: Sixty patients who received our GEM/DOCE protocol between 2007-2017 were identified (51 BCG failures, 9 BCG naïve). This study measured overall treatment success, defined as no recurrence, progression, cystectomy, nor death due to bladder cancer. Kaplan-Meier curves were used to ascertain probability of treatment success. The log-rank test was used to identify factors associated with treatment success.

RESULTS: Sixty patients received treatment with a median follow-up of 14.9 months. All patients completed the induction course with no significant adverse effects. Overall treatment success was $83 \%$ at first surveillance, $69 \%$ at 1 year, and 55\% at 2 years in the entire cohort, and $90 \%$ at first surveillance, $74 \%$ at 1 year, and $56 \%$ at 2 years in the BCG-failure patients. All-cause and bladder-cancer-specific survival were both $97.9 \%$ at 1 year, $85.9 \%$ and $94.6 \%$ respectively at 2 years. Three patients underwent cystectomy at a median of 10.2 months, two of these were secondary to recurrences. Three patients had progression of their disease.
\end{abstract}

CONCLUSIONS: Hyperthermic GEM/DOCE seems to be a well-tolerated salvage regimen that demonstrates a reasonable efficacy and warrants further investigation.

Keywords: Salvage chemotherapy, BCG failure, combination chemotherapy and hyperthermia

\section{INTRODUCTION}

Current guidelines state that disease management

*Correspondence to: Donald L. Lamm, MD, BCG Oncology, 3815 E Bell Rd Ste 1210, Phoenix, AZ 85032, USA. Tel.: +1 602493 6626; Fax: +1 602996 1383; E-mail: dlamm@bcgon cology.com. of non-muscle invasive bladder cancer (NMIBC) should include a transurethral resection of all bladder tumors (TURBT) (with repeat resection in $\mathrm{T} 1$ disease), followed by intravesical Bacillus Calmette- 
Guérin (BCG) immunotherapy for intermediate and high-risk tumor patients [1-3]. Intravesical BCG has been shown to reduce the rate of disease recurrence and disease progression, as well as improve disease-specific survival $[4,5]$. Despite being the gold standard treatment, up to $40 \%$ of individuals with NMIBC do not respond to intravesical BCG therapy [6] and up to $75 \%$ of individuals will develop a new tumor within 5 years [7]. The latest European Association of Urology (EAU) guidelines state that patients facing BCG failure are unlikely to respond to further therapy with BCG. Although the guidelines acknowledge various bladder preservation options in the setting of BCG failure, they classify them as oncologically inferior and present radical cystectomy (RC) as the preferred option $[1,6,8]$. However, many patients prefer bladder preservation after considering the potential morbidity and mortality associated with RC [9], while others are not surgical candidates for various reasons. In this situation, salvage intravesical treatments have become an important option for patients.

Gemcitabine, a nucleoside analog, was shown to be an effective treatment option in NMIBC BCG refractory patients previously [10-12], with a 1-year recurrence-free survival rate for patients of $28 \%$ [11]. Docetaxel, a microtubule inhibitor, has been shown to be effective in a wide range of cancers, including bladder cancer [13]. In one study, patients who failed BCG were given salvage intravesical Docetaxel therapy and the 1 and 2-year recurrence-free survival rates were $45 \%$ and $32 \%$ respectively [14]. Docetaxel therapy has been shown to have even higher efficacy when combined with other chemotherapeutic agents $[13,15]$. Furthermore, hyperthermia has shown to improve bladder preservation rates [16]. In a systematic review looking at intravesical Mitomycin-C (MMC) and hyperthermia, the results showed a 59\% relative reduction in NMIBC recurrence with chemohyperthermia than with MMC alone [16].

In an effort to provide an additional salvage treatment option, a dose-dense, hyperthermic, combination Gemcitabine and Docetaxel chemotherapy treatment (GEM/DOCE) has been offered at our institution since 2007 to patients with NMIBC who failed BCG but sought an alternative to RC. In 2015, Steinberg et al. published the first study of sequential GEM/DOCE as a salvage therapy for recurrent NMIBC after BCG failure in 45 patients, and demonstrated a disease free survival rate of $54 \%$ at 1 year and $34 \%$ at 2 years [15]. Milbar et al. published a similar study in 2017 with 33 patients utilizing the same chemotherapy protocol as Steinberg et al. and demonstrated a DFS rate of $42 \%$ at 1 year and $24 \%$ at 2 years [17]. Although our intravesical chemotherapy protocol is similar to that of Steinberg et al., hyperthermic and dose-dense combination therapy with these specific agents has not been previously studied. We report our institution's experience with our salvage combination Gemcitabine and Docetaxel regimen for patients with NMIBC who failed BCG therapy and were poor surgical candidates for radical cystectomy or desired bladder preservation.

\section{MATERIALS AND METHODS}

\section{Study population and design}

After Institutional Review Board approval was received (protocol number 1607708171), patients who received concentrated intravesical, hyperthermic, GEM/DOCE between 2007-2017 at our institution were identified $(n=60)$ and retrospectively reviewed.

This study measured overall treatment success, defined as no bladder cancer recurrence, no progression to muscle invasion or metastasis, no cystectomy, and no death due to bladder cancer. Time to recurrence, all-cause and bladder-cancer-specific survival rates were also measured. The study also looked at any complications or side effects that occurred as a result of the chemotherapy. Exclusion criteria included lack of significant follow-up and receiving alternative intravesical agents during the course of treatment. Significant follow-up was defined as having a 3-month cystoscopy with cytology to assess response, and any subsequent clinic visits to assess progress.

\section{Gemcitabine/Docetaxel intravesical treatment protocol}

For induction, patients receive 6 weeklyinstillations of the combination intravesical chemotherapy. Patients take sodium bicarbonate (in the form of Alka-Seltzer tablets, 2 tablets the night before the procedure and 2 tablets the morning of the procedure) to alkalinize their urine. They are asked to refrain from drinking any liquids 4 hours prior to the treatment. Sterile water diluent is warmed to $43-45^{\circ} \mathrm{C}$. (A microwave or coffee warmer can be used to heat a water bath in which the water to be used to dilute the drugs is placed. Delivery syringe temperature is tested on inner arm to confirm safety.) The bladder is carefully drained with a 
Foley catheter, and the Foley balloon is filled with 20-40 $\mathrm{mL}$ (depending on bladder capacity) of warm water. $200 \mathrm{mg}$ Gemcitabine in $10 \mathrm{~mL}$ of warm water is instilled and the catheter clamped. With the small volume used, air is used to chase the drug and ensure it is all instilled (amount of air needed depends on size of catheter used, for a $16 \mathrm{Fr}$ catheter $4.5 \mathrm{ml}$ of air is used). Balloon water is exchanged with warm water every 20 minutes as patients are rotated from front to back, and side to side. After one hour, the Gemcitabine is emptied and $20 \mathrm{mg}$ of Docetaxel in $10 \mathrm{~mL}$ of warm water is instilled, and the catheter is removed. The patients are instructed to retain the fluid in their bladder for 120 minutes. Gemcitabine is always given before Docetaxel, because studies have shown the removal of the urothelial barrier by exfoliation with the use of Gemcitabine allows for better taxane penetrance and therefore improved drug efficacy [18].

Maintenance consists of 3 weekly-treatments of the combination chemotherapy at 3 months, 6 months, and 9 months. This maintenance schedule is patterned after the remarkably successful 3 week maintenance schedule for BCG immunotherapy. Each maintenance course follows a surveillance cystoscopy, and can be done on the same day.

\section{Surveillance}

Cystoscopies are performed every 3 months up until 2 years after treatment induction. Afterwards, the cystoscopies are spaced to every 6 months. Bladder washings with cytology are performed with every cystoscopy.

\section{Statistical analysis}

Data was retrospectively collected and stored in a deidentified database. Univariate Cox regression was performed to evaluate for any clinical predictors of recurrence. Kaplan-Meier curves were used to ascertain probability of treatment success in patients categorized by pre-treatment grade (CIS, HG, LG), classification of BCG failure, number of prior BCG induction courses, and number of positive pre-treatment bladder pathology samples. The log rank test was used to identify statistical differences between these respective groups. The above statistical protocols were used to analyze all-cause survival and bladder-cancer-specific survival as well. Statistical analysis and Kaplan-Meier graph generation was done with STATA version 14.

\section{RESULTS}

\section{Cohort demographics}

The 60-patient cohort had a median age at treatment of 73 years (Table 1). Nine (15\%) patients in the cohort were BCG naïve, of whom 6 patients were transplant recipients and immunosuppressed. The remaining 51 patients failed previous BCG therapy. Patients with a BCG failure were further classified by their sub-types: BCG intolerant (disease recurrence after a less than adequate course of therapy is applied due to a serious adverse event or symptomatic intolerance), BCG relapsing (recurrence of disease after achieving a disease-free status by 6 months), or BCG refractory (rapidly recurrent or progressive disease noted at 3 months after diagnosis or persistent disease at 6 months after diagnosis in light of 2 BCG induction courses or induction plus maintenance) [15]. Four (7\%) patients in the cohort were BCG intolerant, 24 (40\%) patients were BCG relapsing, 19 (32\%) patients were BCG refractory, and 4 (7\%) patients who failed BCG could not be further categorized based on the available information from previous records. The median number of prior BCG induction and maintenance courses are 1 (range 0-3) and 1 (range 0-6) respectively.

\section{Treatment tolerance}

Thirty-one patients (52\%) reported experiencing adverse symptoms during their GEM/DOCE treatment course, but only 10 of these patients had symptoms (i.e. UTI) that impacted the treatment schedule with short 1-week delays. All the patients were still able to finish their treatment course. The most common side effects noted were mild fatigue $(20 \%)$, hematuria $(20 \%)$, mild urinary frequency/urgency $(13 \%)$, dysuria (10\%), and nocturia $(7 \%)$.

\section{Treatment success}

Overall treatment success was $83 \%(50 / 60)$ at first surveillance, $69 \%$ at 1 year, and $55 \%$ at 2 years after induction of GEM/DOCE (Fig. 1). The overall median follow-up for the cohort was 14.9 months (range 1.9-89.4 months). In those who failed therapy $(n=21,20$ recurrence, 1 cystectomy not related to recurrence), median time to failure was 6.1 months (range 2.4-21.4 months). Treatment success in those who failed BCG therapy (cohort minus BCG naive) was $88 \%(45 / 51)$ at first surveillance, $74 \%$ at 1 year, and $56 \%$ at 2 years after induction of GEM/DOCE 
Table 1

Demographics of patients who received combination Gemcitabine and Docetaxel for NMIBC

\begin{tabular}{lc}
\hline Variables & $\mathrm{N}=60$ \\
\hline Age at GEM/DOCE induction (median, range) & $73(48-88)$ \\
Gender (male, \%) & $47(68.1)$ \\
Race (Caucasian, \%) & $54(90.0)$ \\
Marital Status (Married, \%) & $48(80.0)$ \\
Smoking Status (yes, \%) & $42(70.0)$ \\
Packs of cigarettes per year (median, range) & $30(1-120)$ \\
Number of BCG Induction Courses & \\
(median, range) & $1(0-3)$ \\
Number of Total BCG Maintenance Courses (median, SD) & $1(0-6)$ \\
Number of Positive Prior Bladder Pathology $(n, \%)$ & \\
1 & $5(8.3)$ \\
2 & $14(20.3)$ \\
3 & $22(36.7)$ \\
$>3$ & $19(31.7)$ \\
Pre-GEM/DOCE NMIBC Stage $(n, \%)$ & \\
CIS Alone & $29(48.3)$ \\
Ta LG & $7(11.7)$ \\
Ta HG & $14(23.3)$ \\
T1 HG & $10(16.7)$ \\
BGC Status $(n, \%)$ & \\
BCG Refractory & $19(31.6)$ \\
BCG Relapse & $24(40.0)$ \\
CG Intolerant & $4(6.7)$ \\
BCG Naive & $9(15.0)$ \\
\hline
\end{tabular}

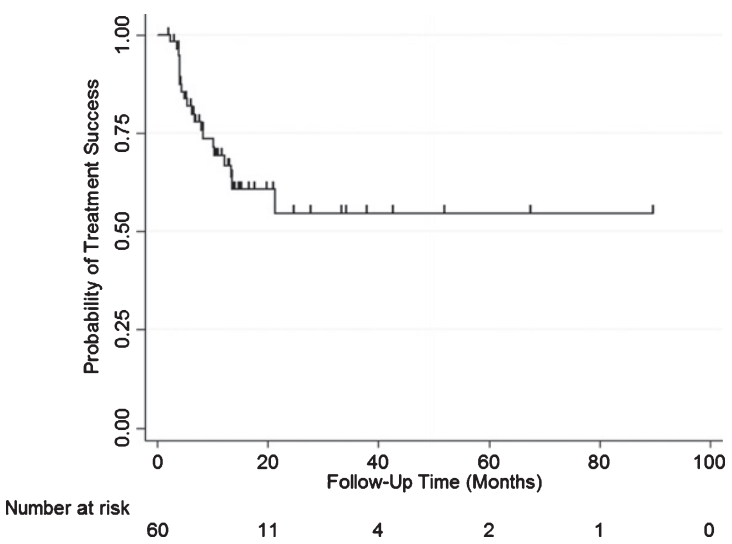

Fig. 1. Kaplan-Meier plot of treatment success with GEM/DOCE in patients with NMIBC $(n=60)$.

(Fig. 2). There was no statistically significant difference in treatment success when the treatment failure group was stratified by pre-chemo stage/grade, BCG failure type, number of prior BCG induction courses, or number of positive prior bladder pathologies (Fig. 3).

\section{Clinical predictors of recurrence}

In order to identify clinical predictors of recurrence after GEM/DOCE treatment, patients were

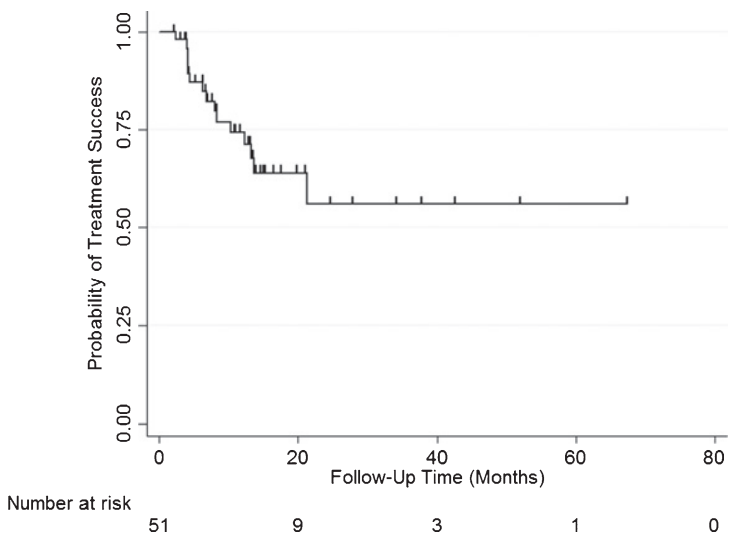

Fig. 2. Kaplan-Meier plot of treatment success with GEM/DOCE in patients with NMIBC who failed BCG therapy $(n=51)$.

stratified by recurrence and their baseline characteristics were analyzed (Table 2). A total of 20 patients $(33 \%)$ had a recurrence of their NMIBC. Patients who underwent more BCG maintenance instillations prior to GEM/DOCE were less likely to recur after receiving GEM/DOCE ( $p=0.048$, HR 0.91). In contrast, prior BCG/IFN treatments increased recurrence $(p=0.046$, HR 8.64). Of note however, there were only 2 patients included in the study who previously received BCG/IFN. Lastly patients who underwent more total GEM/DOCE instillations were less likely 
A

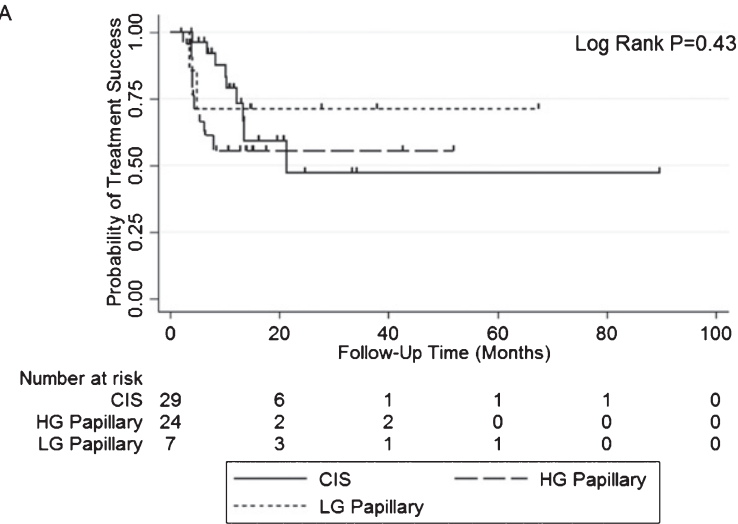

C

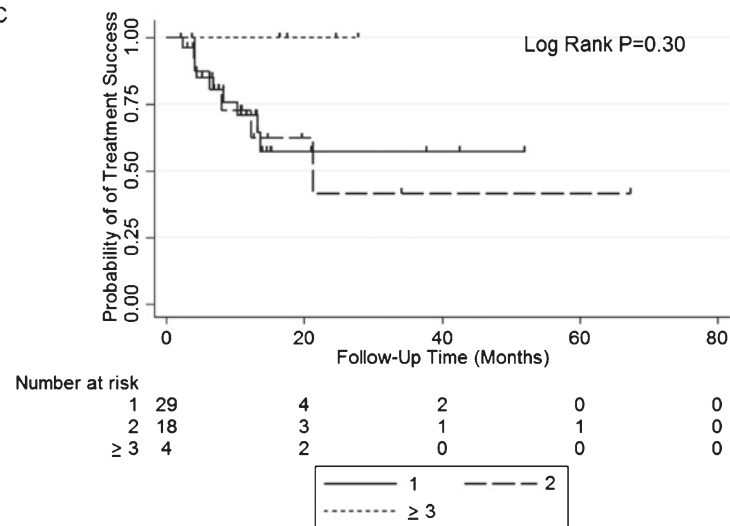

B

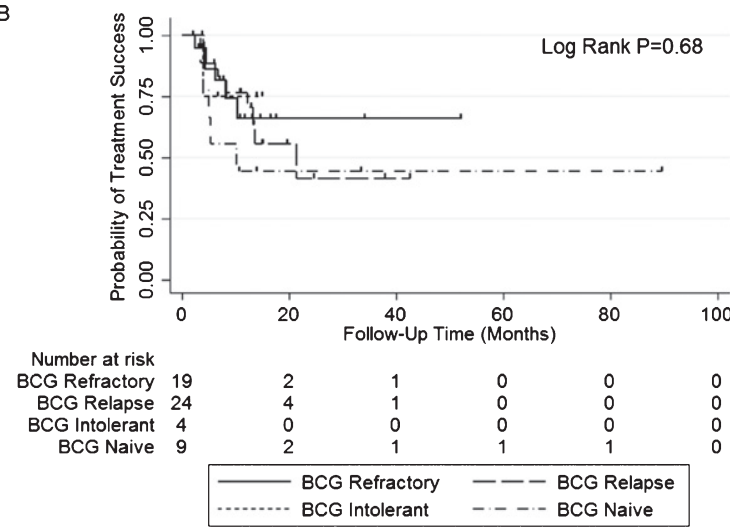

D

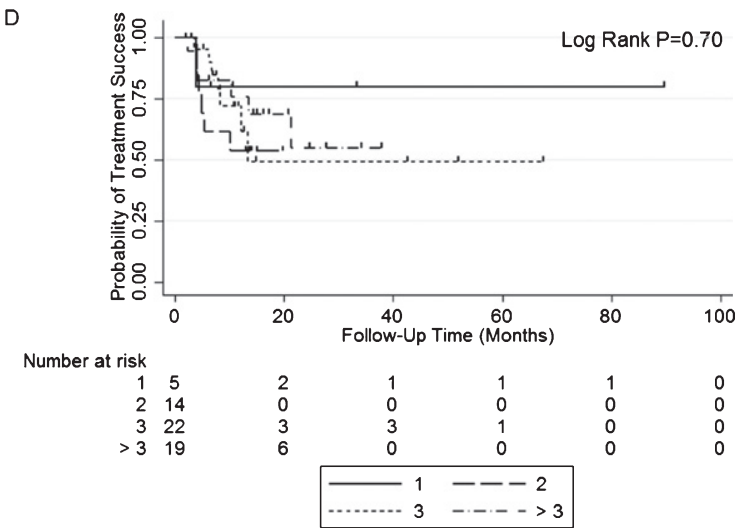

Fig. 3. Kaplan-Meier plots of treatment success with GEM/DOCE in patients with NMIBC stratified by A) Pre-chemo stage/grade; B) Type of BCG failure; C) Number of prior BCG failures; and D) Number of prior positive NMIBC bladder pathologies.

Table 2

Characteristics of patients who received GEM/DOCE for NMIBC stratified by recurrence status

\begin{tabular}{|c|c|c|c|c|}
\hline Variables & $\begin{array}{c}\text { No Recurrence } \\
\quad \mathrm{N}=40\end{array}$ & $\begin{array}{c}\text { Yes Recurrence } \\
\quad \mathrm{N}=20\end{array}$ & $\begin{array}{c}\text { HR } \\
(95 \% \mathrm{CI}) \\
\end{array}$ & $P$-value \\
\hline Age at GEM/DOCE induction (mean, SD) & $74.2(11.0)$ & $69.6(7.21)$ & $0.95(0.91,1.00)$ & 0.064 \\
\hline Gender (male, \%) & $32(80.0)$ & $15(75.0)$ & $0.58(0.21,1.63)$ & 0.31 \\
\hline Race (Caucasian, \%) & $36(90.0)$ & $18(90.0)$ & $1.10(0.25,4.79)$ & 0.89 \\
\hline Marital Status (Married, \%) & $30(75.0)$ & $18(90.0)$ & $2.38(0.55,10.3)$ & 0.24 \\
\hline Smoking Status (yes, \%) & $28(70.0)$ & $14(70.0)$ & $1.40(0.53,3.67)$ & 0.48 \\
\hline Pack-years (mean, SD) & $33.2(27.9)$ & $31.8(32.2)$ & $0.99(0.97,1.02)$ & 0.77 \\
\hline Number of BCG Maintenance Courses (mean, SD) & $1.49(1.78)$ & $0.93(1.27)$ & $0.76(0.53,1.11)$ & 0.16 \\
\hline Number of Total BCG Maintenance Instillations (mean, SD) & $12.9(5.93)$ & $10.5(4.82)$ & $0.91(0.83,0.99)$ & 0.048 \\
\hline Prior BCG/IFN Treatments (yes, \%) & $1(2.50)$ & $1(5.00)$ & $8.64(1.03,71.8)$ & 0.046 \\
\hline Other Prior Treatments (yes, \%) & $8(20.0)$ & $4(20.0)$ & $0.81(0.26,2.45)$ & 0.71 \\
\hline Number of Total BCG and BCG/IFN Maintenance Courses (mean, SD) & $1.51(1.77)$ & $0.93(1.28)$ & $0.76(0.53,1.10)$ & 0.16 \\
\hline Number of Positive Prior Bladder Pathology (mean, SD) & $3.25(1.66)$ & $3.35(2.11)$ & $1.01(0.79,1.26)$ & 0.96 \\
\hline Duration of time for GEM/DOCE Induction (mean, SD) & $6.08(0.65)$ & $6.15(0.59)$ & $1.19(0.65,2.17)$ & 0.56 \\
\hline Number of Total GEM/DOCE Instillations (mean, SD) & $10.8(3.87)$ & 9.55 (3.87) & $0.86(0.76,0.97)$ & 0.015 \\
\hline
\end{tabular}

to recur ( $p=0.015$, HR 0.86$)$. There was no statistical significance noted for age, gender, race, marital status, smoking status, pack-years, number of BCG maintenance courses, other treatments, number of BCG maintenance courses, number of prior positive bladder pathology results, and duration of time (in weeks) for completion of GEM/DOCE induction.

\section{Cystectomies}

Of the 55 potential cystectomy candidates prior to GEM/DOCE, 3 patients underwent cystectomy at a median of 10.2 months (range 6.1-12.3 months) from the time of first GEM/DOCE instillation (Table 3). One patient could not tolerate the dysuria, frequency 
Table 3

Patients treated with GEM/DOCE who underwent a RC

\begin{tabular}{lllll}
\hline$\#$ & $\begin{array}{l}\text { Time to cystectomy } \\
\text { from GEM/DOCE } \\
\text { initiation (months) }\end{array}$ & $\begin{array}{l}\text { Reason for } \\
\text { cystectomy }\end{array}$ & Stage & $\begin{array}{l}\text { BCG Failure } \\
\text { Type }\end{array}$ \\
\hline 1 & 6.1 & $\begin{array}{l}\text { Lack of improvement } \\
\text { in urinary symptoms }\end{array}$ & T0, N0 & BCG Unresponsive \\
2 & 10.2 & $\begin{array}{l}\text { Recurrence } \\
\text { Recurrence }\end{array}$ & $\begin{array}{l}\text { metastatic Progression } \\
\text { Tis, N0 }\end{array}$ & $\begin{array}{l}\text { BCG Relapsing } \\
\text { BCG Relapsing }\end{array}$ \\
\hline
\end{tabular}

A

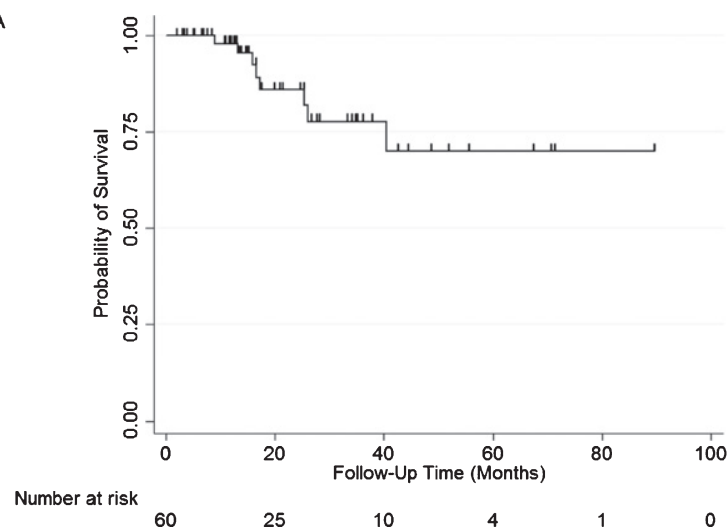

B

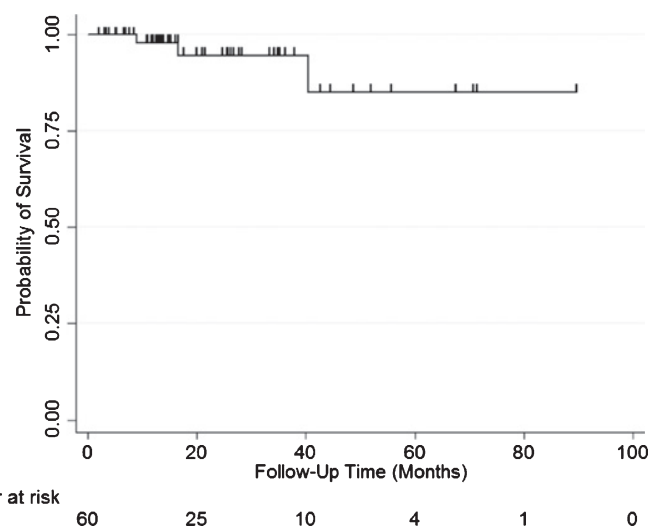

Fig. 4. Kaplan Meier plots of A) All- cause survival and B) Bladder-cancer-specific survival in patients with NMIBC treated with GEM/DOCE.

and nocturia that remained from their initial cancer even after GEM/DOCE induction was completed, and thus chose to undergo a cystectomy. The remaining two patients chose to undergo a cystectomy after they experienced a recurrence.

\section{Survival analysis}

All-cause and bladder-cancer-specific survival were both $97.9 \%$ at 1 year (Fig. 4). At 2 years, all-cause and bladder-cancer-specific survival were $85.9 \%$ and $94.6 \%$ respectively. The first bladdercancer-specific mortality patient, who was not a cystectomy candidate, was found to have muscle invasive disease 2.4 months after GEM/DOCE initiation, and subsequently passed away 9 months after their GEM/DOCE initiation. The second bladdercancer-specific patient had a recurrence at 8.3 months after GEM/DOCE initiation, underwent a cystectomy at 10.2 months at which time he was found to have metastatic disease, and subsequently passedaway at 16.7 months after GEM/DOCE initiation. The third and final bladder-cancer-specific mortality patient underwent surveillance for 20 months after GEM/DOCE and was found not to recur, afterwards the patient chose to stop surveillance and eventu- ally passed away from metastatic disease progression at 40.4 months after GEM/DOCE initiation at an advanced age.

\section{DISCUSSION}

Patients with NMIBC who fail BCG therapy remain a complicated population to treat. Current recommendations by the European Association of Urology (EAU) state that in patients who fail BCG, radical cystectomy $(\mathrm{RC})$ still remains the preferred option $[1,6,8]$. However, RC has significant risks and comorbidities associated with it [9]. Although Steinberg and Milbar published their studies on GEM/DOCE first, to our knowledge, this is the first study looking at administering GEM/DOCE with an almost $3 \times$ concentrated docetaxel dose, while utilizing the benefits of hyperthermia during instillation of the chemotherapy agents. All 60 patients in our study were able to complete the full induction course of GEM/DOCE and none had severe adverse events related to the treatment. This supports the findings of previous GEM/DOCE studies which have found this combination of intravesical chemotherapy to be well-tolerated and relatively safe $[15,17]$. 
Our treatment protocol was successful in $69 \%$ of patients at 1 year, which is in line with the success rates found by Steinberg (54\% at 1 year) and Milbar ( $42 \%$ at 1 year) $[15,17]$. The same is true for our success at 2 years of $55 \%$, which is also in line with the findings of Steinberg (34\% at 2 years) and Milbar (24\% at 2 years) $[15,17]$. Our success rates were higher when specifically looking at the BCG failure population with 1 year and 2 year success rates of $74 \%$ and $56 \%$ respectively.

When compared to other chemotherapeutic agents that have been studied as treatment options for NMIBC, our protocol had higher success rates than those of Mitomycin-C (MMC) which had a 1 year RFS rate of $65 \%$ and a 3 year RFS rate of $19 \%$ [19]. In 2013, the results of a SWOG Phase II trial with single-agent intravesical Gemcitabine in patients who had failed at least 2 courses of BCG showed the 1 year recurrence-free survival rates for patients with a complete response to the treatment was $28 \%$, and the 2 year recurrence-free survival rate was $21 \%$ [11]. The study used Gemcitabine at a dose of 2,000 mg/100 mL and then followed with monthly maintenance therapy for a year. In another study, patients who failed BCG were given singleagent intravesical Docetaxel therapy with a maximum dose of $75 \mathrm{mg} / 100 \mathrm{ml} \mathrm{(3/8} \mathrm{of} \mathrm{the} \mathrm{concentration} \mathrm{used}$ in our protocol). The 1 and 2 year recurrence-free survival rates were $45 \%$ and $32 \%$ respectively [14]. Additionally, our protocol compares favorably with Gemcitabine and MMC combination therapy which had a 1 year RFS rate of $48 \%$ and a 2 year RFS rate of $38 \%$ [20].

It is important to note that $3(5 \%)$ patients who completed GEM/DOCE underwent progression of their disease at a median of 8.3 months (range 2.4-20+ months). One patient progressed to higher grade disease and the remaining two patients progressed to muscle invasive bladder cancer (one of whom elected for a cystectomy). Despite these risks associated with pursuing salvage chemotherapy, we feel the success rates of our protocol and the higher quality of life afforded to our patients who were able to avoid RC, both warrant further investigation into GEM/DOCE.

In 2014, the Food and Drug Administration (FDA) and American Urological Association (AUA) made recommendations that new bladder cancer therapies should have an initial complete response rate of $40 \%-50 \%$ at 6 months and a durable response rate of at least $30 \%$ for 18-24 months (with the lower bound of the $95 \%$ confidence interval (CI) excluding
$20 \%$ ) to be clinically meaningful [21]. We propose that our success rates are above these cutoffs, including the lower bound of our $95 \% \mathrm{CI}$ at 24 months, which was $36 \%$. Thus, we argue further investigation of GEM/DOCE in a prospective, controlled trial is justified. Additionally, our data shows GEM/DOCE could have a role as a first-line therapy in patients with NMIBC. In our study 9/60 (15\%) high-risk patients were BCG naïve and underwent GEM/DOCE as their first-line treatment. Five $(56 \%)$ of these patients recurred, $3(33 \%)$ of whom recurred with high-grade disease. Both the 1 year and 2 year success rates in the BCG naïve group were $44 \%$. Considering the current shortages of BCG in the United States, GEM/DOCE could potentially serve as an alternative to BCG therapy in order to prevent delay of treatment and thus warrants further study as a first-line treatment.

This study is limited by its retrospective design and having no control group to compare the GEM/DOCE results to. Another limitation is the moderate cohort size, which can reduce the power of the statistical analysis. Additionally, the study included 68\% men and over $90 \%$ Caucasian patients, which is a limited distribution among gender and ethnicity. Another potential limitation of this study is that we cannot compare our hyperthermia technique with the previous favorable data reported with the Synergo and Mitomycin C. Furthermore, we do not believe but cannot exclude a selection bias in favor of GEM/DOCE. In the earlier years of the retrospective study other salvage chemotherapy regimens were used, including doxorubicin/mitomycin and various combinations including docetaxel and gemcitabine. Combinations with doxorubicin or MMC were used in $24 \%$ of patients, and multiple dual combinations in $27 \%$ of patients in the initial years. Since GEM/DOCE appeared to be both more effective and less toxic than other combinations (with the earlier preliminary data showing $34.8 \%$ recurrence for GEM/DOCE versus $65 \%$ other salvage regimens), other combinations and regimens were abandoned for new patients. When available, patients eligible for clinical trials such as CG0070 or TMX-101-003 were offered such treatment.

In conclusion, hyperthermic combination Gemcitabine and Docetaxel appears to be a well-tolerated salvage regimen that demonstrates a reasonable efficacy and meets the criteria for new therapies for NMIBC set by the FDA and AUA in 2014. Our results further confirm and show success rates higher than previously published studies on GEM/DOCE in 
NMIBC. As such, further investigation to optimize a protocol for patients who fail or are not candidates for BCG and do not want a RC is warranted.

\section{ACKNOWLEDGMENTS}

None.

\section{FUNDING}

The authors report no funding.

\section{AUTHOR CONTRIBUTIONS}

MYR and DLL contributed to the study design, acquisition of data, analysis and interpretation of data, and drafted the manuscript; PK contributed to the analysis and interpretation of the data; JCT and DLM contributed to the acquisition of the data. All authors critically reviewed the final manuscript and gave final approval.

\section{CONFLICT OF INTEREST}

The authors have no conflict of interest to report.

\section{REFERENCES}

[1] Babjuk M, Böhle A, Burger M, Capoun O, Cohen D, Compérat EM, et al. EAU guidelines on non-muscleinvasive urothelial carcinoma of the bladder: Update 2016. Eur Urol. 2017;71:447-61.

[2] Chang SS, Boorjian SA, Chou R, Clark PE, Daneshmand $\mathrm{S}$, Konety BR, et al. Diagnosis and treatment of nonmuscle invasive bladder cancer: AUA/SUO guideline. J Urol. 2016;196:1021-9.

[3] Brausi M, Witjes JA, Lamm D, Persad R, Palou J, Colombel $\mathrm{M}$, et al. A review of current guidelines and best practice recommendations for the management of nonmuscle invasive bladder cancer by the International Bladder Cancer Group. J Urol. 2011;186:2158-67.

[4] Böhle A, Bock P. Intravesical bacille calmette-guérin versus mitomycin $\mathrm{c}$ in superficial bladder cancer: Formal meta-analysis of comparative studies on tumor progression. Urology. 2004;63:682-6.

[5] Sylvester RJ, van der MEIJDEN APM, Lamm DL. Intravesical bacillus Calmette-Guerin reduces the risk of progression in patients with superficial bladder cancer: A meta-analysis of the published results of randomized clinical trials. J Urol. 2002;168:1964-70.

[6] Zlotta AR, Fleshner NE, Jewett MA. The management of BCG failure in non-muscle-invasive bladder cancer: An update. Can Urol Assoc J. 2009;3:S199-205.

[7] Kamat AM, Colombel M, Sundi D, Lamm D, Boehle A, Brausi $\mathrm{M}$, et al. BCG-unresponsive non-muscle-invasive bladder cancer: Recommendations from the IBCG. Nat Rev Urol. 2017;14:244-55.
[8] O'Donnell MA, Boehle A. Treatment options for BCG failures. World J Urol. 2006;24:481-7.

[9] Lawrentschuk N, Colombo R, Hakenberg OW, Lerner SP, Månsson W, Sagalowsky A, et al. Prevention and management of complications following radical cystectomy for bladder cancer. Eur Urol. 2010;57:983-1001.

[10] Dalbagni G, Russo P, Bochner B, Ben-Porat L, Sheinfeld J, Sogani P, et al. Phase II trial of intravesical gemcitabine in bacille calmette-guérin-refractory transitional cell carcinoma of the bladder. J Clin Oncol. 2006;24:2729-34.

[11] Skinner EC, Goldman B, Sakr WA, Petrylak DP, Lenz H-J, Lee CT, et al. SWOG S0353: Phase II trial of intravesical gemcitabine in patients with nonmuscle invasive bladder cancer and recurrence after 2 prior courses of intravesical bacillus Calmette-Guérin. J Urol. 2013;190:1200-4.

[12] Perdonà S, Di Lorenzo G, Cantiello F, Damiano R, De Sio M, Masala $D$, et al. Is gemcitabine an option in BCG-refractory nonmuscle-invasive bladder cancer? A single-arm prospective trial. Anticancer Drugs. 2010;21:101-6.

[13] McKiernan JM, Masson P, Murphy AM, Goetzl M, Olsson CA, Petrylak DP, et al. Phase I trial of intravesical docetaxel in the management of superficial bladder cancer refractory to standard intravesical therapy. J Clin Oncol. 2006;24:3075-80.

[14] Barlow LJ, McKiernan JM, Benson MC. The novel use of intravesical docetaxel for the treatment of non-muscle invasive bladder cancer refractory to BCG therapy: A single institution experience. World J Urol. 2009;27:331-5.

[15] Steinberg RL, Thomas LJ, O'Donnell MA, Nepple KG. Sequential intravesical gemcitabine and docetaxel for the salvage treatment of non-Muscle invasive bladder cancer. B1 Cancer IOS Press. 2015;1:65-72.

[16] Lammers RJM, Witjes JA, Inman BA, Leibovitch I, Laufer M, Nativ $\mathrm{O}$, et al. The role of a combined regimen With intravesical chemotherapy and hyperthermia in the management of non-muscle-invasive bladder cancer: A systematic review. Eur Urol. Elsevier; 2011;60:81-93.

[17] Milbar N, Kates M, Chappidi MR, Pederzoli F, Yoshida $\mathrm{T}$, Sankin A, et al. Oncological outcomes of sequential intravesical gemcitabine and docetaxel in patients with nonmuscle invasive bladder cancer. B1 cancer (Amsterdam, Netherlands). 2017;3:293-303.

[18] Pandey R, Jackson JK, Liggins R, Mugabe C, Burt HM. Enhanced taxane uptake into bladder tissues following coadministration with either mitomycin $\mathrm{C}$, doxorubicin or gemcitabine: Association to exfoliation processes. BJU Int John Wiley \& Sons, Ltd (10.1111). 2018;122:898-908.

[19] Malmström PU, Wijkström H, Lundholm C, Wester K, Busch C, Norlén BJ. 5-year followup of a randomized prospective study comparing mitomycin $\mathrm{C}$ and bacillus Calmette-Guerin in patients with superficial bladder carcinoma. Swedish-Norwegian Bladder Cancer Study Group. J Urol. 1999;161:1124-7.

[20] Lightfoot AJ, Breyer BN, Rosevear HM, Erickson BA, Konety BR, O'Donnell MA. Multi-institutional analysis of sequential intravesical gemcitabine and mitomycin C chemotherapy for non-muscle invasive bladder cancer. Urol Oncol Semin Orig Investig. 2014;32:35.e15-35.e19.

[21] Jarow JP, Lerner SP, Kluetz PG, Liu K, Sridhara R, Bajorin $\mathrm{D}$, et al. Clinical trial design for the development of new therapies for nonmuscle-invasive bladder cancer: Report of a food and drug administration and american urological association public workshop. Urology. 2014;83: $262-5$. 